\title{
An atomic finite element model for biodegradable polymers. Part 1. Formulation of the finite elements
}

Andrew Gleadall ${ }^{\star a}$, Jingzhe Pan ${ }^{b}$, Lifeng Ding ${ }^{c}$, Marc-Anton Kruft ${ }^{d}$ and David Curco ${ }^{e}$

a Manufacturing and Process Technologies, Faculty of Engineering, University of Nottingham, University Park, Nottingham, NG7 2RD, UK

${ }^{\mathrm{b}}$ Department of Engineering, University of Leicester, Leicester, LE1 7RH, UK

c A555, Science Building, Xi'an Jiaotong-Liverpool University 111, Ren'ai Road Dushu

Lake Higher Education Town, Suzhou Industrial Park, Suzhou, Jiangsu, 215123, China.

d Corbion Purac Biomaterials, P.O. Box 21, 4200 AA Gorinchem, The Netherlands

e Chemical Engineering Department, University of Barcelona, Martí i Franquès 1, 08028, Barcelona, Spain

* Corresponding author. Tel: +44(0)115 823 2507; Fax: +44(0)115 951 3800; E-mail: andrew.gleadall@nottingham.ac.uk;

\section{Abstract}

Molecular dynamics (MD) simulations are widely used to analyse materials at the atomic scale. However, MD has high computational demands, which may inhibit its use for simulations of structures involving large numbers of atoms such as amorphous polymer structures. An atomic-scale finite element method (AFEM) is presented in this study with significantly lower computational demands than MD. Due to the reduced computational demands, AFEM is suitable for the analysis of Young's modulus of amorphous polymer structures. This is of particular interest when studying the degradation of bioresorbable polymers, which is the topic of an accompanying paper. AFEM is derived from the interatomic potential energy functions of an MD force field. The nonlinear MD functions were adapted to enable static linear analysis. Finite element formulations were derived to 
represent interatomic potential energy functions between two, three and four atoms. Validation of the AFEM was conducted through its application to atomic structures for crystalline and amorphous poly(lactide).

Key words: Biodegradable polymers, Young's modulus, atomic simulations, finite element analysis, molecular dynamics.

\section{Introduction}

Biodegradable polymers such as poly(lactide), poly(glycolide) and poly(caprolactone) have been used in medical applications for several decades. However, their mechanical properties are not fully understood due to the complex nature of both polymer theory and degradation. Recent studies modelling mechanical property degradation have begun to increase knowledge in the area [1,2]. Further insights into biodegradable polymer properties and degradation mechanisms may be achieved through atomic analysis. This opportunity has generated interest in the development of molecular dynamics (MD) force fields for poly(lactide) [3-6]. These force fields describe the interactions between atoms by relating the atomic coordinates to interatomic potential energy.

Ding et al. [1] studied the chain scission of a polyethylene atomic structure through MD simulations. It was not possible for Ding et al. [1] to use a complex molecular structure, such as poly(lactide), due to the high computational requirements of MD simulations. This limitation has led to research into more computationally efficient simulation methods. The procedure used in MD simulations is as follows: (i) calculate the instantaneous forces applied by the atoms to one another; (ii) calculate the atomic acceleration that results from the forces; (iii) calculate the speed and distance travelled by each atom in a discrete time period; (iv) calculate the new atom positions and repeat from step i. This 
computationally intense process can be simplified by using molecular statics, in which the simulation can be considered to include a damping force that gradually removes energy from the system until the atoms have zero velocity. Although molecular statics simulations require less computational power than molecular dynamics, amorphous polymers still present a computational challenge. Especially when repeated simulations are required, for example when analysing a polymer during degradation.

To enable the analysis of more complex structures, or to enable a greater number of simulations, several authors have developed more computationally efficient method for atomic analysis [7-10]. Liu et al. [9, 10] developed an atomic-scale finite element method (AFEM) in which the molecular dynamics force field is represented by finite elements. Their method offered significant reductions in computational demands versus the conjugate gradient method in molecular mechanics. For nonlinear potential energy functions, an iterative procedure was required in order to minimise the total potential energy in a structure. Wang et al. $[7,8]$ also developed atomistic finite elements to represent the interaction between atoms through finite element analysis. The elements they developed represent the polymer as a chain of elastic rods connected to each other by elastic joints, which facilitate angular potential energy terms to be considered. Again, an iterative procedure was used in the simulations. The work of Liu et al. $[9,10]$ and Wang et al. [7, 8] demonstrate the value of atomistic finite element methods. However, due to the iterative nature of their methods, the computation benefits over MD are limited.

This study presents a new atomic finite element method (AFEM), in which nonlinear interatomic potential energy functions are adapted to enable static linear analysis. Simulations are completed in a single step as opposed to numerous iterations. The reduced computational demands permit the analysis of large atomic structures, including amorphous poly(lactide). This simplification is possible if one is only interested in very 
small displacement of atoms from their equilibrium positions, such as when calculating Young's modulus of a polymer. Atomic finite elements are specifically formulated to represent all atomic interactions in the PLAFF2 [5] molecular dynamics force field for poly(lactide). In this first paper of a two-part series, the AFEM simulation technique is developed and demonstrated for crystalline and amorphous poly(lactide) structures. In the second paper [11] the effect of polymer chain scission on the degradation of Young's modulus is analysed. Such analysis has not previously been conducted for an atomic model of a biodegradable polymer. The AFEM simulation results are used to develop a numerical model for Young's modulus change of degrading polymers.

\section{Molecular dynamics polymer force field}

In molecular dynamics, the interactions between atoms are described by force fields that relate atomic coordinates to potential energy. McAliley developed the PLAFF2 force field specifically for poly(lactide) and supplied it as supplementary information with his thesis [5]. This force field is used as a basis for the AFEM approach presented in this study. This section gives a brief overview in Eqs. 1 - 8 of the atomic interactions described by the force field. Further details can be found in McAliley's thesis [5] and in the manual of the MD software Gromacs [12]. Fig. 1 shows a four-atom section of a polymer chain. Several different forms of atomic interaction are shown. In the force field, each type of interaction is represented by a potential energy function. Potential energy is calculated for covalently bonded atoms based on:

- interatomic separation (the bond-stretch potential energy);

- the angle between adjacent bonds (the bond-angle potential energy;

- and rotation of the polymer chain about a bond (the bond-dihedral potential energy). 
The figure also shows how potential energy is associated with the interatomic separation of atoms that are not covalently bonded (the nonbond potential energy). These potential energy forms combine to represent the overall atomic interaction in a material. Although the AFEM approach presented in this study is based on a specific force field, it simply translates to a wide range of force fields for different materials.

Atomic simulations of material properties typically consider the potential energy of a representative unit cell of polymer, which repeats to infinity in all directions. The total potential energy of the unit cell, $V_{M D}\left(\mathrm{~kJ} \mathrm{~mol}^{-1}\right)$, according to the PLAFF2 force field is given in Eq. 1 as

$$
V_{M D}=\sum_{\substack{\text { bonds } \\ i, j}} V_{b}+\sum_{\substack{\text { angles } \\ i, j, k}} V_{a}+\sum_{\substack{\text { dihedrals } \\ i, j, k, l}} V_{d}+\sum_{\substack{\text { nonbonds } \\ i, j}} V_{n b}-F u
$$

which is the sum of potential energies for all bond-stretch, $V_{b}$, bond-angle, $V_{a}$, bonddihedral, $V_{d}$, and nonbonded, $V_{n b}\left(\right.$ all $\left.\mathrm{kJ} \mathrm{mol}^{-1}\right)$, atomic interactions. The term $F u$ is the applied work due to an externally applied force, $F(\mathrm{~N})$, and the displacement where $F$ is applied, $u(\mathrm{~nm})$. In molecular statics simulations, the atomic coordinates are found such that $V_{M D}$ is minimised. The minimisation of potential energy results in the atomic configuration that the force field suggests is likely to exist in a real material. When an overall force is applied to the material and results in a strained atomic configuration, the increase in potential energy is used to calculate Young's modulus.

Bond-stretch potential energy, $\boldsymbol{V}_{b}\left(\mathrm{~kJ} \mathrm{~mol}^{-1}\right)$, of two covalently bonded atoms is given by

$$
V_{b}=\frac{1}{2} k_{b}\left(r_{i j}-r_{0}\right)^{2}
$$

in which $k_{b}\left(\mathrm{~kJ} \mathrm{~mol}^{-1} \mathrm{~nm}^{-2}\right)$ is the bond-stretch force constant, $r_{i j}(\mathrm{~nm})$ is the atomic separation, and $r_{0}(\mathrm{~nm})$ is the equilibrium atomic separation. 
Bond-angle potential energy, $V_{a}\left(\mathrm{~kJ} \mathrm{~mol}^{-1}\right)$, is calculated as

$$
V_{a}=\frac{1}{2} k_{a}\left(\theta-\theta_{0}\right)^{2}
$$

in which $k_{a}\left(\mathrm{~kJ} \mathrm{~mol}^{-1} \mathrm{rad}^{-2}\right)$ is the bond-angle force constant, $\theta(\mathrm{rad})$ is the angle between atoms $\mathrm{i}, \mathrm{j}, \mathrm{k}$, and the equilibrium angle is $\theta_{0}(\mathrm{rad})$.

Bond-dihedral potential energy, $V_{d}\left(\mathrm{~kJ} \mathrm{~mol}^{-1}\right)$, in the force field includes both proper and improper dihedrals which are shown schematically in Fig. 1. Proper dihedrals consider atoms which are covalently bonded in series. Their potential energy is related to rotation about the covalent bond between the middle two atoms. Improper dihedrals in PLAFF2 consider four atoms of which three are all covalently bonded to a central atom. These can be considered to be in the form of a pyramid, as shown in Fig. 1. The potential energy function is effectively related to rotation about one of the bottom edges of the pyramid. For a set of four atoms, i, j, k, I, covalently bonded in series, the dihedral angle, $\Phi(\mathrm{rad})$, is defined as the angle between the plane of atoms $\mathrm{i}, \mathrm{j}, \mathrm{k}$, and the plane of atoms $\mathrm{j}, \mathrm{k}, \mathrm{l}$. The angle is defined according to the IUPAC/IUB convention such that an angle of zero corresponds to the cis configuration with atoms $i$ and I on the same side. The force field utilises the Ryckaert-Bellemans dihedral potential energy, $V_{d(\text { Ryckaert-Bellemans) }}$ $\left(\mathrm{kJ} \mathrm{mol}{ }^{-1}\right)$, according to

$$
V_{d(\text { Ryckart-Bellemans })}=\sum_{n=0}^{5} C_{n}\left(\cos \left(\phi-180^{\circ}\right)\right)
$$

in which $C_{n}\left(\mathrm{~kJ} \mathrm{~mol}^{-1}\right)$ are six Ryckaert-Bellemans coefficients. The force field also considers a periodic dihedral function for which the periodic dihedral potential energy, $V_{d(\text { periodic) }}\left(\mathrm{kJ} \mathrm{mol}^{-1}\right)$, is given by

$$
V_{d(\text { periodic })}=\frac{1}{2} k_{d}\left(1+\cos \left(n_{\phi} \phi-\phi_{0}\right)\right)^{2}
$$


in which $k_{d}\left(\mathrm{~kJ} \mathrm{~mol}^{-1} \mathrm{rad}^{-2}\right)$ is the periodic dihedral force constant, $\Phi_{0}(\mathrm{rad})$ is the phase shift and $n_{\Phi}$ (no units) is the multiplicity which dictates the number of peaks in the potential energy for one complete rotation of the bond.

Nonbond potential energy, $V_{n b}\left(\mathrm{~kJ} \mathrm{~mol}^{-1}\right)$, is calculated as the sum of Lennard-Jones potential energy, $V_{\text {Lennard-Jones }}\left(\mathrm{kJ} \mathrm{mol}^{-1}\right)$, and Coulomb interaction potential energy, $V_{\text {coulomb }}\left(\mathrm{kJ} \mathrm{mol}^{-1}\right)$, as given by

$$
V_{n b}=V_{\text {Lennard-Jones }}+V_{\text {Coulomb }} \text {. }
$$

The Lennard-Jones potential energy accounts for the long range attractive van der Waals interactions and the short range Pauli repulsion due to overlapping electron orbitals. It can be calculated as

$$
V_{\text {Lennard-Jones }}=4 \sqrt{\varepsilon_{i i} \varepsilon_{j j}}\left(\left(\frac{\sqrt{\sigma_{i i} \sigma_{i j}}}{r_{i j}}\right)^{12}-\left(\frac{\sqrt{\sigma_{i i} \sigma_{i j}}}{r_{i j}}\right)^{6}\right)
$$

in which $r_{i j}(\mathrm{~nm})$ is the interatomic separation, $\varepsilon_{i i}$ and $\varepsilon_{j j}\left(\mathrm{~kJ} \mathrm{~mol}^{-1}\right)$ are the Lennard-Jones well-depth parameters for atoms $\mathrm{i}$ and $\mathrm{j}$ respectively, and $\sigma_{i i}$ and $\sigma_{i j}(\mathrm{~nm})$ are the LennardJones radii for atoms $\mathrm{i}$ and $\mathrm{j}$ respectively. The Coulomb interaction potential energy accounts for the attraction or repulsion of atoms due to their permanent charge and is given by

$$
V_{\text {Coulomb }}=\frac{1}{4 \pi \varepsilon_{0}}\left(\frac{q_{i} q_{j}}{r_{i j}}\right)
$$

in which $\varepsilon_{0}\left(\mathrm{~F} \mathrm{~m}^{-1}\right)$ is the electric constant, $q_{i}$ and $q_{j}$ (units of elementary charge) are electric charges for atoms $\mathrm{i}$ and $\mathrm{j}$ respectively.

In the PLAFF2 force field, the nonbond potential energy function does not apply to atoms that are separated by just one or two covalent bonds since the repulsive forces become too great. It is assumed that the bond-stretch and bond-angle potential energy terms 
adequately represent the short-range quantum mechanical atomic interactions. For nonbonds between atoms that are separated by exactly three covalent bonds, the nonbond repulsion may still be too strong and hence their potential energy contribution is halved. These exclusions and reductions of potential energies are frequently implemented in molecular dynamics simulations. 


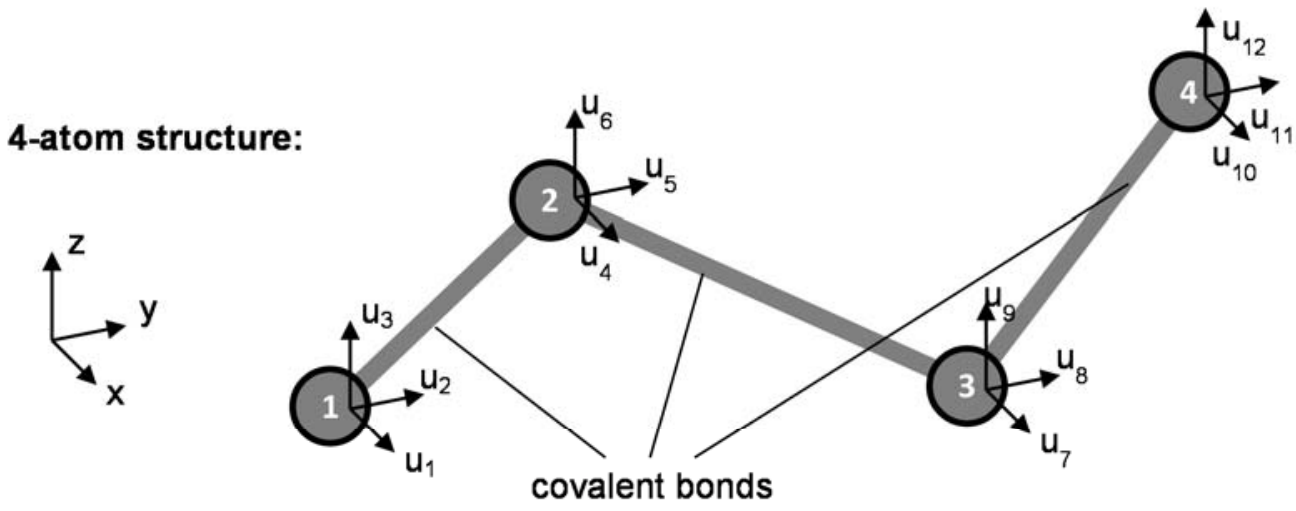

Individual elements:

$3 x$ bond-stretch elements:
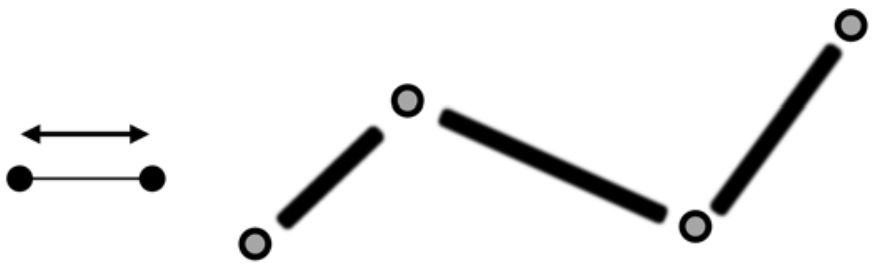

$2 x$ bond-angle elements:
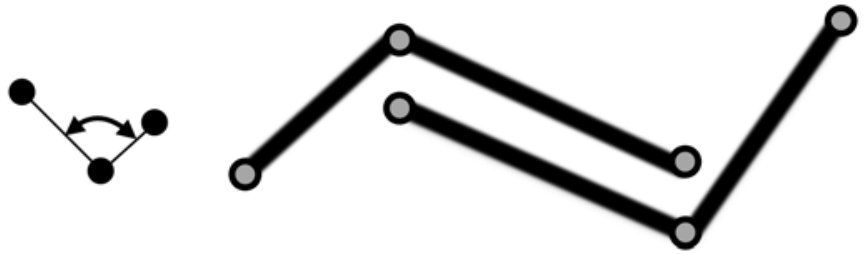

$1 \mathrm{x}$ bond-dihedral element:

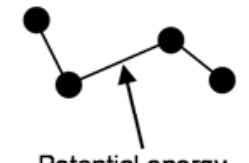

Potential energy related to rotation about this bond

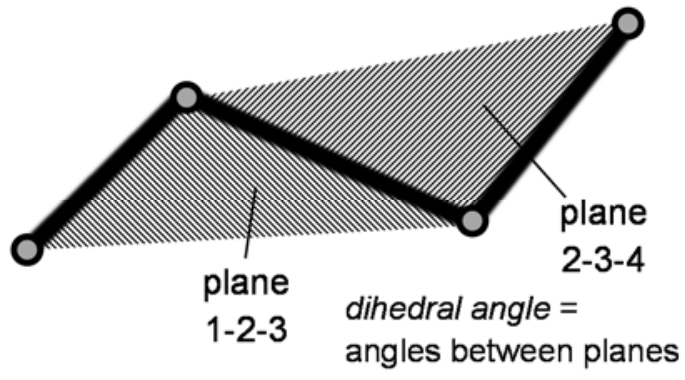

\section{Additional elements:}

Improper dihedral elements:

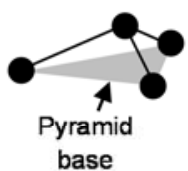

$$
\text { Resist flattening }
$$
of the pyramid
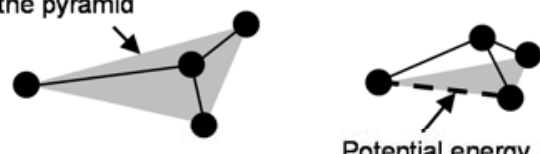

Potential energy related to rotation

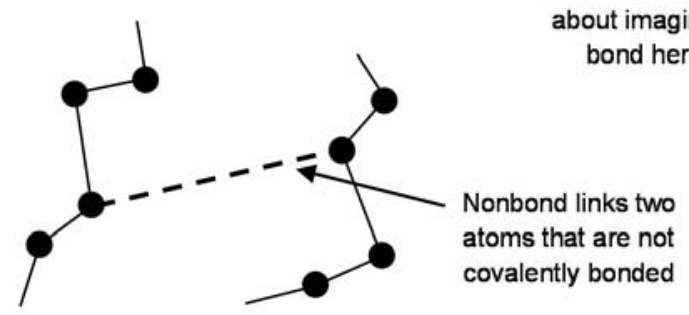

Fig. 1 Schematic of the interatomic potential energy terms: bond-stretch, bond-angle, bond-dihedrals, which are divided into proper and improper dihedrals, and nonbonds. 


\section{Adaptation of nonlinear MD potential energies terms for AFEM}

The AFEM approach presented in this study is based on the MD force field PLAFF2, which was discussed in the previous section. The bond-dihedral and nonbond potential energy terms in the force field are not suitable for static linear analysis. They require iterative simulation techniques, which have much greater computational demands. To enable static linear analysis, the total potential energy given in Eq. 1 is adapted here. The adapted total potential energy of the unit cell, $V_{M D_{-} F E M}\left(\mathrm{~kJ} \mathrm{~mol}^{-1}\right)$, is given by Eq. 9 as

$$
V_{M D_{-} F E M}=\sum_{\substack{\text { bonds } \\ i, j}} V_{b}+\sum_{\substack{\text { angles } \\ i, j, k}} V_{a}+\sum_{\substack{\text { dihedrals } \\ i, j, k, l}} V_{d_{-} F E M}+\sum_{\substack{\text { nonbonds } \\ i, j}} V_{n b_{-} F E M}-F u
$$

in which $V_{d_{-} F E M}\left(\mathrm{~kJ} \mathrm{~mol}^{-1}\right)$ is the adapted bond-dihedral potential energy and $V_{n b_{-} F E M}(\mathrm{~kJ}$ $\mathrm{mol}^{-1}$ ) is the adapted nonbond potential energy. As with MD simulations, the total potential energy is minimised in order to find the atomic displacements due an externally applied force. The molecular dynamics bond-stretch and bond-angle potential energy terms in the PLAFF2 force field take the harmonic form as given in Eqs. 2 and 3. The bond-dihedral and nonbond potential energy terms take more complex forms. They are adapted here to also take a harmonic form.

Finite elements are derived in this study to represent each of the potential energy terms in the force field. The potential energy of each individual finite element, $V_{e l}$ is $\left(\mathrm{kJ} \mathrm{mol}^{-1}\right)$, is

$$
V_{e l}=\frac{1}{2} k_{e l} d_{e l}{ }^{2} \text {. }
$$

In Eq. $10, k_{e l}$ is the force constant $\left(\mathrm{kJ} \mathrm{mol}^{-1} \mathrm{~nm}^{-2}\right.$ for linear displacement or $\mathrm{kJ} \mathrm{mol}^{-1} \mathrm{rad}^{-2}$ for angular displacement). The term $d_{e l}$ is the change in length $(\mathrm{nm})$ for bond-stretch and nonbond finite elements, or change in angle (rad) for bond-angle and bond-dihedral finite elements. The subscript el is set to:

- $\quad b$, to represent bond-stretch finite elements 
- $a$, to represent bond-angle finite elements

- d_FEM, to represent bond-dihedral finite elements

- and $n b \_F E M$, to represent nonbond finite elements.

The double derivative of potential energy $V_{e l}$ in Eq. 10 with respect to change in length or angle $d_{e l}$ gives finite element stiffness $k_{e l}$ according to

$$
\frac{d^{2}\left(V_{e l}\right)}{d\left(d_{e l}\right)^{2}}=k_{e l} \text {. }
$$

Since the PLAFF2 force field uses a harmonic form of bond-stretch and bond-angle potential energy, $k_{e l}$ takes the values $k_{b}$ and $k_{a}$ which are explicitly given in the force field for use in Eqs. 2 and 3. For the bond-dihedral and nonbond potential energy terms however, the values of $k_{d_{-} F E M}$ and $k_{n b_{-} F E M}$ are not explicitly given in PLAFF2. They are derived here for use in the harmonic potential energy in AFEM by twice differentiating the PLAFF2 potential energy terms for bond-dihedrals and nonbonds with respect to length or angle. This represents an assumption that the element stiffness does not change significantly during deformation, which is appropriate for studies of very small displacements.

Eqs. 12 and 13 give the double derivatives of the two bond-dihedral potential energy terms used in the PLAFF2 force field with respect to the dihedral angle $\Phi$ :

$$
\begin{aligned}
& \frac{d^{2} V_{d(\text { Ryckaert }- \text { Bellemans })}}{d \Phi^{2}}=-C 1 \cos (\Phi)+2 C 2 \sin (\Phi)^{2} \\
& -2 C 2 \cos (\Phi)^{2}+6 C 3 \cos (\Phi) \sin (\Phi)^{2}-3 C 3 \cos (\Phi)^{3} \\
& +12 C 4 \cos (\Phi)^{2} \sin (\Phi)^{2}-4 C 4 \cos (\Phi)^{4} \\
& +20 C 5 \cos (\Phi)^{3} \sin (\Phi)^{2}-5 C 5 \cos (\Phi)^{5} \\
& \frac{d^{2} V_{d(\text { periodic })}}{d \Phi^{2}}=-k_{d} \cos \left(n \Phi-\Phi_{0}\right) n^{2} .
\end{aligned}
$$


These derivatives give $k_{d_{-} F E M}$, the bond-dihedral finite element stiffness based on the atomic coordinates. The double derivative of the nonbond potential energy terms used in the PLAFF2 force field is

$$
\frac{d^{2} V_{n b}}{d r_{i j}^{2}}=\frac{1}{2} \frac{q_{i} q_{j}}{\pi \varepsilon_{0} r_{i j}^{3}}+4 \sqrt{\varepsilon_{i} \varepsilon_{j}}\left(\frac{156 \sigma_{i i}^{6} \sigma_{i j}^{6}}{r_{i j}^{14}}-\frac{42 \sigma_{i i}^{3} \sigma_{i j}^{3}}{r_{i j}^{8}}\right)
$$

which gives that value of nonbond finite element stiffness, $k_{n b_{-} F E M}$, based on atomic separation. Fig. 2 plots potential energy, along with its first and second derivatives, versus the interatomic separation for two atoms according to the PLAFF2 force field. The figure considers a carbonyl carbon atom and a carbonyl oxygen atom. Similar trends exist for other atom pairs. Eqs. 6 - 8 are used to plot the potential energy and its derivatives. The parameters for these equations are taken from the PLAFF2 force field. The first derivative gives the interatomic force that the nonbonded atoms apply to one another. The second derivative gives the atomic finite element stiffness, which indicates resistance to changes in interatomic separation. At short interatomic separations the atoms apply a positive force to each other, which indicates repulsion due to electron orbital overlap. At the equilibrium distance the interatomic force is zero because the atoms' attraction and repulsion perfectly balance. As the interatomic separation increases further, the atoms apply an attractive interatomic force to each other. At the interatomic separation highlighted by the vertical dashed line in the figure, the nonbond effectively breaks because further elongation of the nonbond results in less force being required to sustain the separation. The structure only remains stable due to interactions with other atoms. The finite element stiffness changes from a positive to a negative value at the vertical dashed line. For the AFEM simulations used to analyse the poly(lactide) polymer structure in this study, nonbond elements with negative stiffness are considered to represent broken bonds and therefore are not included in the simulations. A similar trend occurs for dihedrals but as opposed to nonbonds, where there is a threshold interatomic separation above which stiffness is negative, there are angular ranges in 
which the dihedral stiffness is negative. In these ranges, the dihedral potential energy function represents an atomic state in which changes to the dihedral angle are not resisted. Therefore bond-dihedral atomic finite elements with a negative stiffness are also excluded from the simulations.

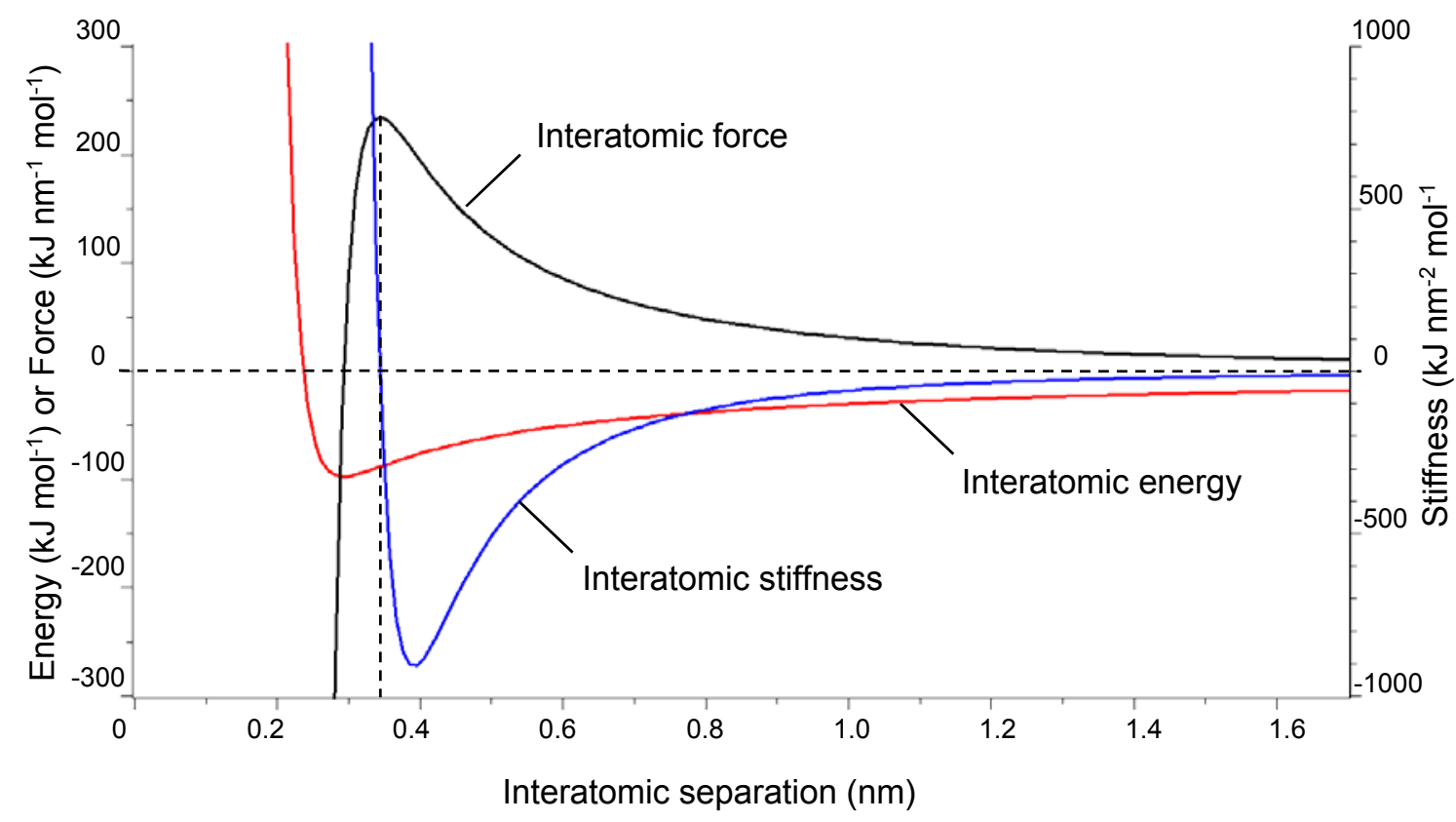

Fig. 2 The PLAFF2 nonbond potential energy is shown for a nonbond between a two atoms in poly(lactide). The single and double derivatives with respect to interatomic separation indicate interatomic force and finite element stiffness respectively.

\section{Formulation of the atomic finite elements}

This section presents the formulation of the atomic finite elements for bond-stretch, bondangle, bond-dihedral and nonbond potential energies. The finite elements are derived from the theory of minimum potential energy as discussed in the previous section. To minimise total potential energy, the differential of the potential energy sum with respect to atomic displacements must equal zero. The Direct Stiffness Method is commonly used to set up finite element simulations. In the Direct Stiffness Method, the individual contribution of each finite element to the overall differential is identified. These 
contributions are calculated here for each potential energy type by differentiating the potential energy of individual finite elements with respect to atomic displacements.

\subsection{Bond-stretch and nonbond AFEM elements}

The bond-stretch and nonbond AFEM elements take the same form as linear elastic spring finite elements. They are derived in Eqs. 15 - 20 using the same approach that that is applied later for the more complex bond-angle and bond-dihedral finite elements. Fig. 3 shows a bond-stretch element with an initial length of $L_{A B}$ before displacement. Total elongation of the element, $\Delta L_{A B}$, is given by

$$
\Delta L_{A B}=\bar{u}_{B} \cdot \bar{r}_{A B}-\bar{u}_{A} \cdot \bar{r}_{A B} \cdot
$$

where $\bar{u}_{A}$ is the vector displacement of atom $A, \bar{u}_{B}$ is the vector displacement of atom $B$ and $\bar{r}_{A B}$ is the vector from atom $A$ to atom $\mathrm{B}$. It is necessary to consider $\Delta L_{A B}$ in terms of the individual Cartesian atomic displacements $u_{1}$ to $u_{6}$ to enable differentiation of Eq. 10 . The term $\alpha_{i}$ is used to indicate the effect of the ith atomic displacement, $u_{i}$, on the overall element elongation, such that total elongation of the element can be described by

$$
\Delta L_{A B}=\sum_{i=1-6} \alpha_{i} u_{i}
$$

This is the sum of all the atomic displacements multiplied by their individual effects on overall element elongation. The six $\alpha_{i}$ terms can be found by differentiating Eq. 15 by the six $u_{i}$ terms according to

$$
\alpha_{i}=\frac{d\left(\Delta L_{A B}\right)}{d\left(u_{i}\right)} .
$$

The interatomic potential energy of the element, is found by substituting $\Delta L_{A B}$ into Eq. 10 as $d_{e l}$ to give

$$
V_{e l}=\frac{1}{2} k_{e l}\left(\sum_{i=1-6} \alpha_{i} u_{i}\right)^{2} .
$$


Eq. 18 is differentiated with respect to $u_{i}$, when minimising total potential energy for AFEM. This differentiation gives the element stiffness matrix that is used in the Direct Stiffness Method. The ith linear equation that results from the differentiation can be written in matrix form as

$$
\frac{d V_{e l}}{d u_{i}}=k_{e l} \alpha_{i}\left[\begin{array}{llllll}
\alpha_{1} & \alpha_{2} & \alpha_{3} & \alpha_{4} & \alpha_{5} & \alpha_{6}
\end{array}\right]\left[\begin{array}{c}
u_{1} \\
u_{2} \\
u_{3} \\
u_{4} \\
u_{5} \\
u_{6}
\end{array}\right] .
$$

The ith row of the element stiffness matrix is therefore given by $k_{e} / \alpha_{i}[\alpha]$. The full $6 \times 6$ element stiffness matrix is therefore

$$
k_{e l}\left[\begin{array}{cccc}
\alpha_{1} \alpha_{1} & \alpha_{1} \alpha_{2} & \ldots & \alpha_{1} \alpha_{6} \\
\alpha_{1} \alpha_{2} & \alpha_{2} \alpha_{2} & \ldots & \alpha_{2} \alpha_{6} \\
\vdots & \vdots & \ddots & \vdots \\
\alpha_{1} \alpha_{6} & \alpha_{2} \alpha_{6} & \cdots & \alpha_{6} \alpha_{6}
\end{array}\right]
$$

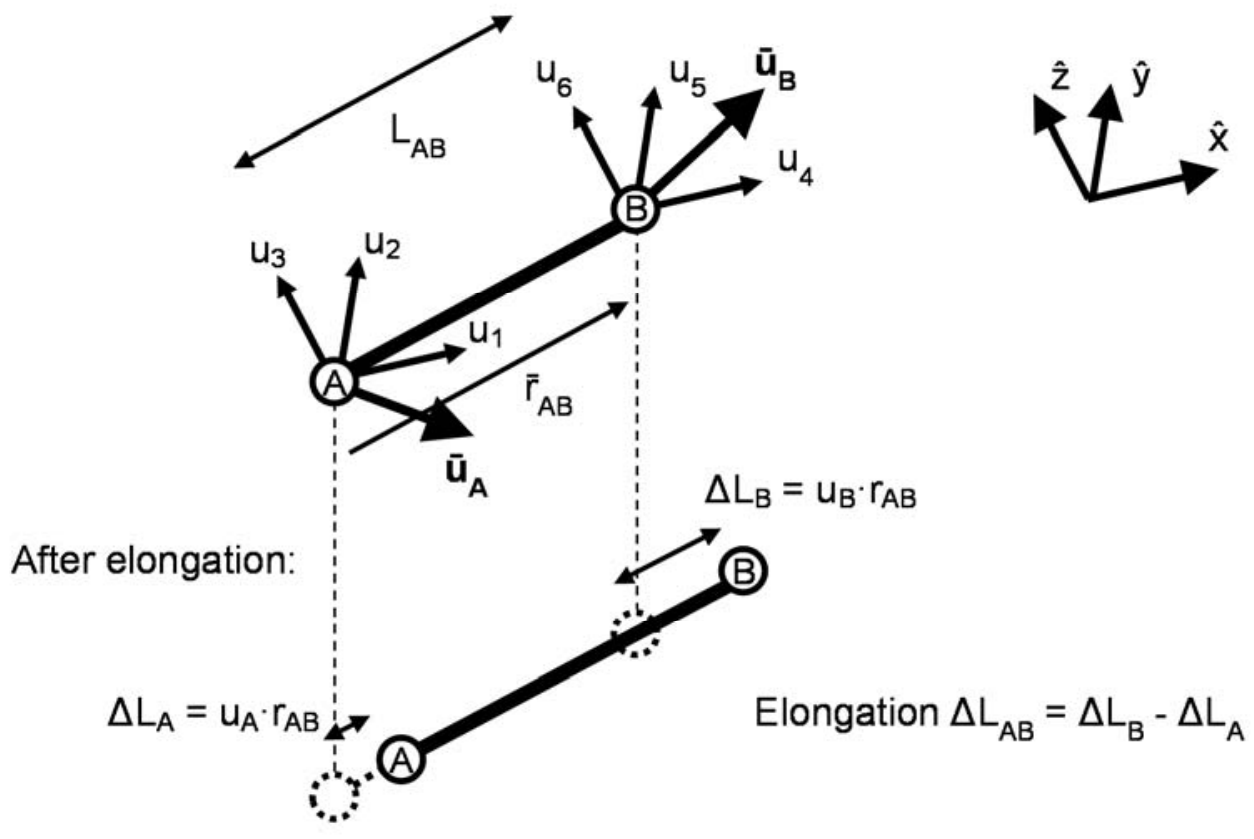


Fig. 3 Schematic of a bond-stretch element in AFEM containing 2 atoms. The displacements of both atoms contribute to overall elongation of the element.

\subsection{Bond-angle AFEM element}

Fig. 4 shows a bond-angle finite element for which change to the angle between three atoms is considered. As with the bond-stretch element above, the change in bond-angle must be formulated in terms of atomic displacements in order to derive the element stiffness matrix. This derivation is described here in Eqs. 21 and 22. The total change in bond-angle in terms of the vector atomic displacements is given by

$$
\Delta \theta=\Delta \theta_{C}-\Delta \theta_{A}=\left(\frac{\bar{u}_{C} \cdot \hat{n}_{C}}{\left|\bar{r}_{B C}\right|}-\frac{\bar{u}_{B} \cdot \hat{n}_{C}}{\left|\bar{r}_{B C}\right|}\right)-\left(\frac{\bar{u}_{A} \cdot \hat{n}_{A}}{\left|\bar{r}_{A B}\right|}-\frac{\bar{u}_{B} \cdot \hat{n}_{A}}{\left|\bar{r}_{A B}\right|}\right)
$$

in which all terms are defined in Fig. 4. As with the bond stretch element formulation above, the effect of each atomic displacement on the bond-angle is calculated by differentiating Eq. 21 with respect to each displacement according to

$$
\alpha_{i}=\frac{d(\Delta \theta)}{d\left(u_{i}\right)} .
$$

The element stiffness matrix is then calculated in exactly the same way as for the bondstretch element in Eqs. 18 - 20. The matrix takes the same form of $\left[\alpha_{i} \alpha_{j}\right]$ except it is $9 \mathrm{x}$ 9 in size.

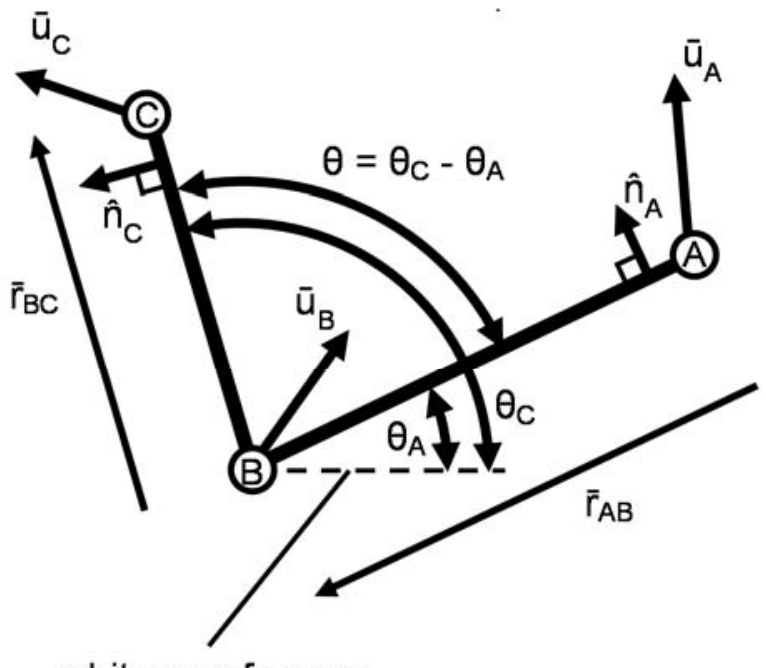

arbitrary reference 
Fig. 4 Schematic of a bond-angle element for 3 atoms. The element is viewed normal to the plane containing all three atoms.

\subsection{Development of the bond-dihedral AFEM element}

Fig. 5 shows a bond-dihedral element which relates the change in the dihedral angle to increase in potential energy. For four atoms, $A, B, C, D$, the bond-dihedral potential energy in the MD force field considers the dihedral angle, $\Phi$, which is defined as the angle between the $A B C$ and $B C D$ planes. The deformation is similar to a mechanical torsion spring as shown in the bottom left of the figure. Fig. 6 shows a dihedral element from several viewpoints, which aid the formulation of the element stiffness matrix. Fig. 6 (a) shows the dihedral element from a similar view to Fig. 5. Fig. 6 (b) shows a view along the vector from atoms $B$ to $C$. This viewpoint demonstrates that changes in the dihedral angle due to deformation of the bond-dihedral element may be expressed as

$$
\Delta \phi=\Delta \phi_{D}-\Delta \phi_{A} .
$$

For small displacements, the change in angle $\Phi_{A}$ in radians due to the vector displacement $\bar{u}_{A}$ of atom $\mathrm{A}$ is

$$
\Delta \phi_{A}=\frac{\bar{u}_{A} \cdot \hat{n}_{A}}{L_{A_{-} n}}
$$

in which $L_{A_{-} n}$ is the perpendicular distance of atom A from the vector $\bar{r}_{B C}$ as shown in Fig.

6 (c). Similarly, the change in angle $\Phi_{D}$ due to displacement of atom $D$ is

$$
\Delta \phi_{D}=\frac{\bar{u}_{D} \cdot \hat{n}_{D}}{L_{D_{-} n}}
$$

where $L_{D_{-} n}$ is defined in Fig. $6(\mathrm{~d})$. 


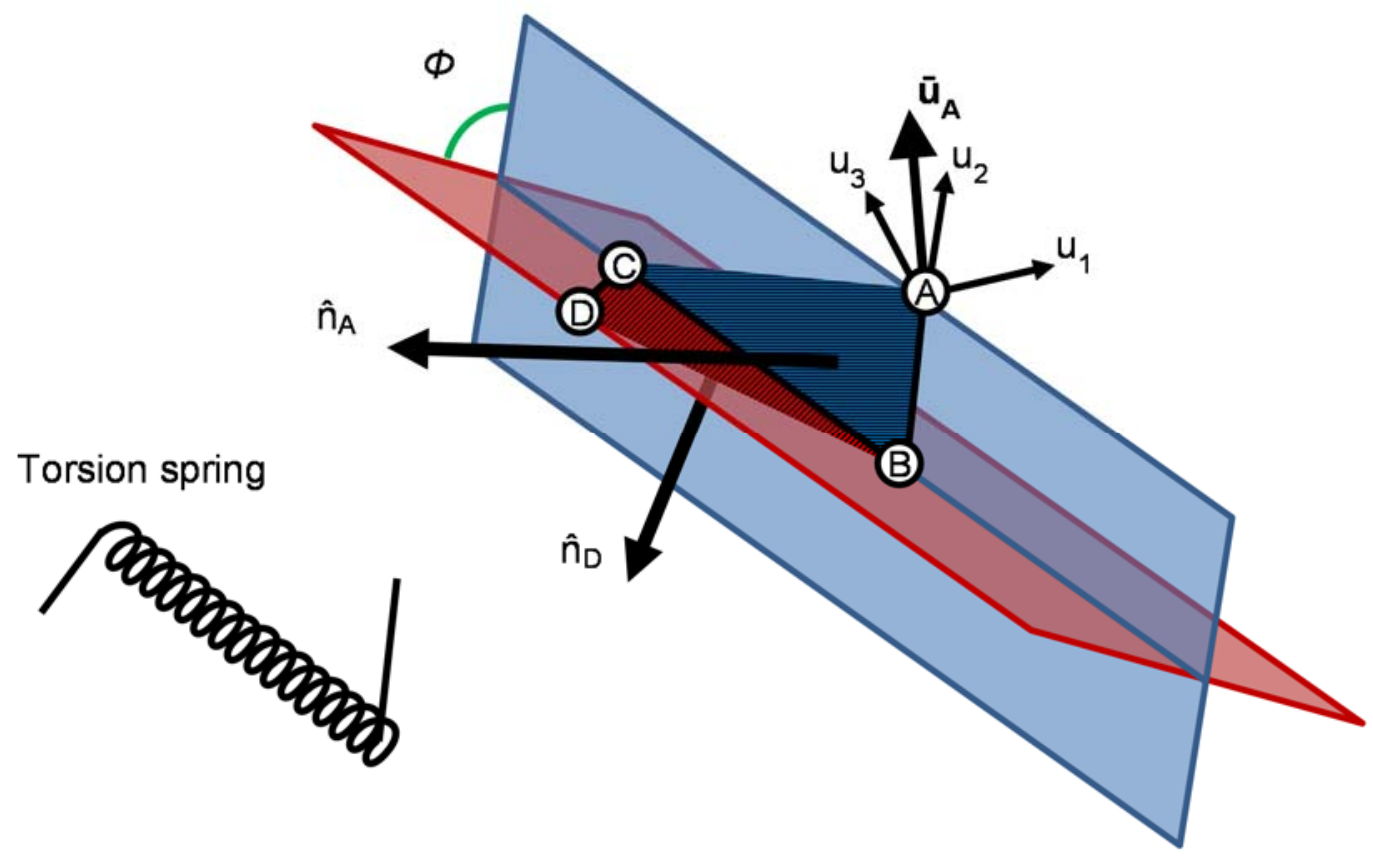

Fig. 5 Schematic of a bond-dihedral element for 4 atoms. Changes to the dihedral angle $\Phi$ between the planes of atoms A, B, C and B, C, D are considered in AFEM.

a) general view

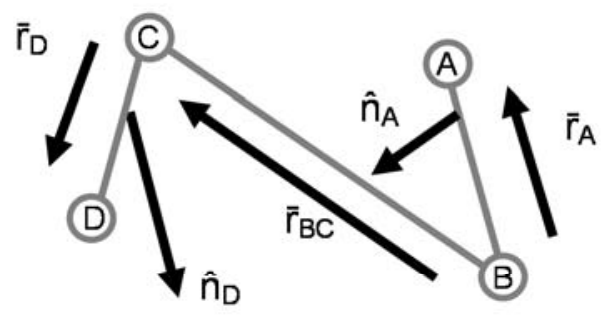

c) view along $\hat{n}_{A}$

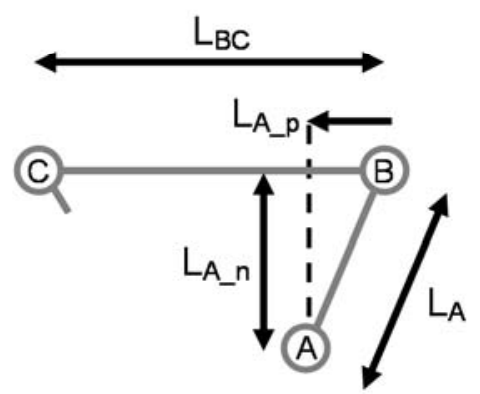

b) view along $\bar{r}_{\mathrm{BC}}$

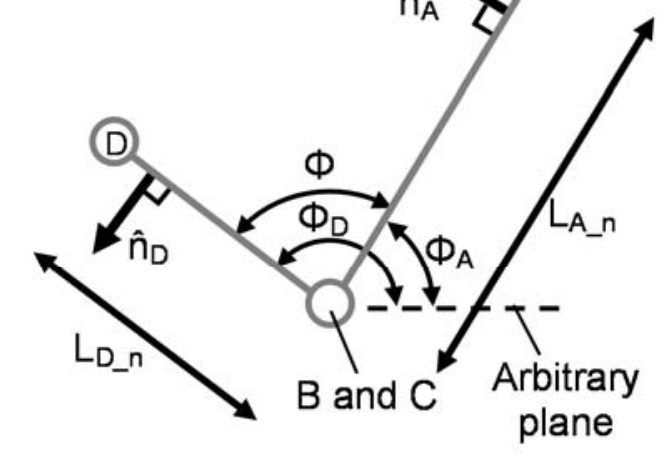

d) view along $\hat{n}_{D}$

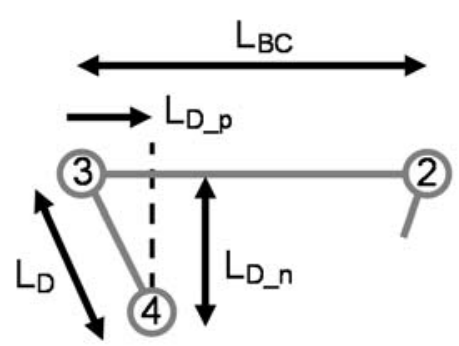

Fig. 6 Definitions of lengths, vectors and angles that are used in the differentiation of the bond-dihedral element for the purpose of minimisation of total potential energy. 
For atoms $\mathrm{B}$ and $\mathrm{C}$, the relationship between their atomic displacement and change in dihedral angle is complicated by the fact that their displacement leads to a change in the vector $\bar{r}_{B C}$. Fig. 7 (a) shows a view along $\bar{r}_{B C}$ with atoms $A$, B and $\mathrm{C}$ all in the horizontal plane. The arbitrary plane used previously in Fig. 6 (b) is chosen here to contain atoms A, B and $C$ so the angle $\Phi_{A}$ is equal to zero in Fig. 7 (a). The horizontal component of any displacement of atom B in Fig. 7 (a) has no effect on the angle $\Phi_{A}$. This is because the plane $A B C$ is unaffected by atomic displacement within the plane. Only the $\hat{n}_{A}$ component of the displacement of atom $B$, given by $\bar{u}_{B} \cdot \hat{n}_{A}$, affects the angle. This effect is demonstrated in Fig. 7 (b) - (d). Fig. 7 (b) shows a perpendicular view of Fig. 7 (a), with atoms A, B and C still in the horizontal plane. Fig. 7 (c) shows a displacement of atom B and Fig. 7 (d) shows an equivalent view to Fig. 7 (a) but along the new $\bar{r}_{B C}$ (after displacement of atom B). As can be seen in Fig. 7 (d), the change in vector $\bar{r}_{B C}$ due to $\bar{u}_{B}$ can be considered to produce an effective displacement of atom A; this is because in the view along the new vector $\bar{r}_{B C}$, the position of atom $\mathrm{A}$ is different than when viewing down the original vector $\bar{r}_{B C}$. The effective displacement of atom A can be substituted into Eq. 24 to identify the change in angle $\Phi_{A}$. Displacement $\bar{u}_{B}$ therefore affects angle $\Phi_{A}$ according to

$$
\Delta \phi_{A}=-\frac{\bar{u}_{B} \cdot \hat{n}_{A}}{L_{A_{-} n}}\left(\frac{L_{B C}-L_{A_{-} P}}{L_{B C}}\right)
$$

in which the terms in brackets are the effective displacement of atom $A$ due to $\bar{u}_{B}$. The terms $L_{B C}$ and $L_{A \_p}$ are shown in Fig. 7. The distance $L_{A \_p}$ is directional and has a sign convention such that it is positive when the angle $A B C$ is less than $90^{\circ}$. In a similar manner, the change in angle $\Phi_{A}$ due to displacement $\bar{u}_{C}$ of atom $C$ is calculated as

$$
\Delta \phi_{A}=-\frac{\bar{u}_{C} \cdot \hat{n}_{A}}{L_{A_{-} n}}\left(\frac{L_{A_{-} P}}{L_{B C}}\right)
$$

and the change in angle $\Phi_{D}$ due to displacements $\bar{u}_{B}$ and $\bar{u}_{C}$ respectively are 


$$
\Delta \phi_{D}=-\frac{\bar{u}_{B} \cdot \hat{n}_{D}}{L_{D_{-} n}}\left(\frac{L_{D_{-} P}}{L_{B C}}\right)
$$

and

$$
\Delta \phi_{D}=-\frac{\bar{u}_{C} \cdot \hat{n}_{D}}{L_{D_{-} n}}\left(\frac{L_{B C}-L_{D_{-} P}}{L_{B C}}\right) .
$$

As with $L_{A \_p}$, the sign convention for $L_{D \_p}$ is that it is positive when the angle BCD is less than $90^{\circ}$. Aside from $L_{A_{\_} p}$ and $L_{D_{-} p}$ all lengths refer to absolute values. The six relationships for angular displacement in Eqs. 24 - 29 combine according to Eq. 23 to give overall angular displacement as

$$
\begin{aligned}
& \Delta \phi=\Delta \phi_{D}-\Delta \phi_{A}= \\
& \left(\frac{\bar{u}_{D} \cdot \hat{n}_{D}}{L_{D_{-} n}}-\frac{\bar{u}_{B} \cdot \hat{n}_{D}}{L_{D_{-} n}}\left(\frac{L_{D_{-} P}}{L_{B C}}\right)-\frac{\bar{u}_{C} \cdot \hat{n}_{D}}{L_{D_{-} n}}\left(\frac{L_{B C}-L_{D_{-} P}}{L_{B C}}\right)\right) \\
& -\left(\frac{\bar{u}_{A \cdot} \cdot \hat{n}_{A}}{L_{A_{-} n}}-\frac{\bar{u}_{C} \cdot \hat{n}_{A}}{L_{A_{-} n}}\left(\frac{L_{A_{-} P}}{L_{B C}}\right)-\frac{\bar{u}_{B} \cdot \hat{n}_{A}}{L_{A_{-} n}}\left(\frac{L_{B C}-L_{A_{-} P}}{L_{B C}}\right)\right)
\end{aligned}
$$

Each of the vector atomic displacements $\bar{u}_{A}, \bar{u}_{B}, \bar{u}_{C}, \bar{u}_{D}$ in Eq. 30 consist of three Cartesian atomic displacements. As demonstrated for bond-stretch and bond-angle finite elements above, Eq. 30 can be written in terms of the 12 atomic displacements $u_{1}$ to $u_{12}$. And the differentials of Eq. 30 with respect to those Cartesian displacements combine to form a $12 \times 12$ element stiffness matrix, as demonstrated in Eqs. 16 - 20. 
a) initial view along $\overline{\mathrm{r}}_{\mathrm{BC}}$

Direction A

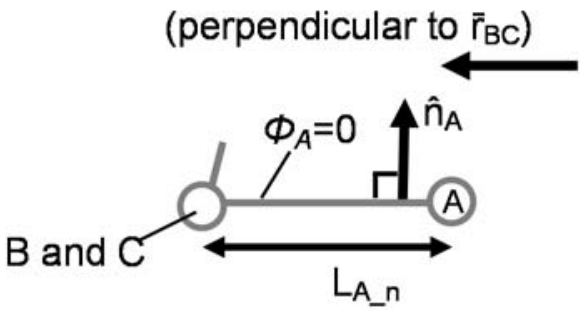

c) the same view as in (b) after displacement of atom $B$

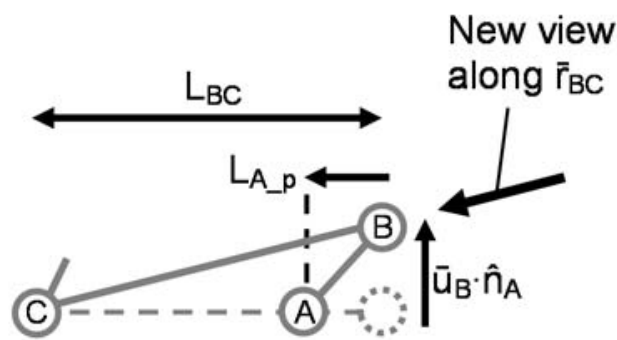

b) initial view along

"Direction A" in (a)

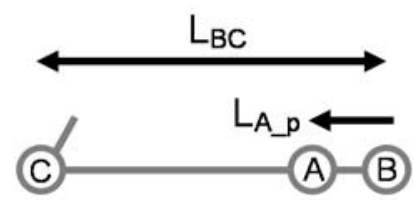

d) the equivalent view to (a) but along the new $\bar{r}_{\mathrm{BC}}$ after displacement of atom $B$

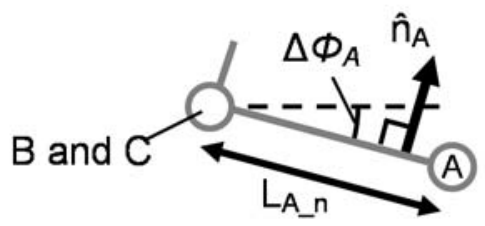

Fig. 7 Schematic of the change in angle $\Phi_{A}$ due to a displacement of atom B while atoms $\mathrm{A}$ and $\mathrm{C}$ remain stationary.

\section{AFEM program setup and polymer structures}

The AFEM simulations import atomic coordinates from molecular dynamics polymer structure files. The coordinates are given for a central unit cell which repeats periodically in $\mathrm{x}, \mathrm{y}$ and $\mathrm{z}$ directions to infinity. Periodic boundary conditions are used in MD and AFEM simulations. A nonbond cut-off distance of $0.95 \mathrm{~nm}$ is used since this value is frequently used in MD simulations. The repeats of this central unit cell are called images. The crystalline structure used in this study was proposed by Sasaki and Asakura [13]. Fig. 8 (a) shows the unit cell of the crystalline structure. The unit cell is repeated once in the $x$, $y$, and $z$ directions for clarity in the figures here. The eight unit cells contain eight helical chains aligned in the $z$ direction, each with 20 repeat polymer units. The overall size is $2.13 \times 1.23 \times 5.78 \mathrm{~nm}$. For simulation of amorphous poly(lactide), the atomic structure 
used was provided as supplementary information with McAliley's thesis [5]. The amorphous unit cell is shown in Fig. 8 (b). It contains three amorphous chains, each consisting of 500 poly repeat units, and is in the form of a cube of side lengths 5.6098 $\mathrm{nm}$. The chains have a molecular weight of $36000 \mathrm{~g} \mathrm{~mol}^{-1}$. Each AFEM simulation of the crystalline structure took less than 5 minutes on a personal computer. Each amorphous simulation for 13500 atoms took approximately 3 hours when utilising 16 processors of a supercomputer. The AFEM simulations were conducted in MATLAB. The computation times could have been significantly improved through the use of an efficiency-optimised linear equation solver.

Young's modulus is calculated based on the strain energy sum for all AFEM elements. For a unit cell of continuous material that is uniaxially strained, the strain energy stored in the material, $U$, is equal to the work that is applied to the material. It is given by

$$
U=\frac{1}{2} F u
$$

where $F$ is the force applied to the unit cell boundary and $u$ is the cell elongation that results from the applied force. The value of strain energy calculated in the AFEM simulations is used to determine $F$. Young's modulus is calculated as stress divided by strain through the values of $F, u$, the unit cell length and the cross-sectional area of the unit cell perpendicular to the applied force. 
a) Crystalline structure

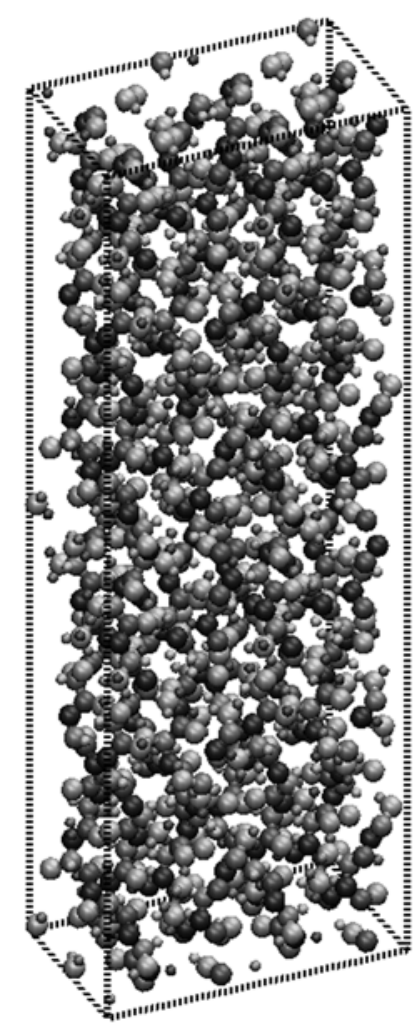

b) Amorphous structure

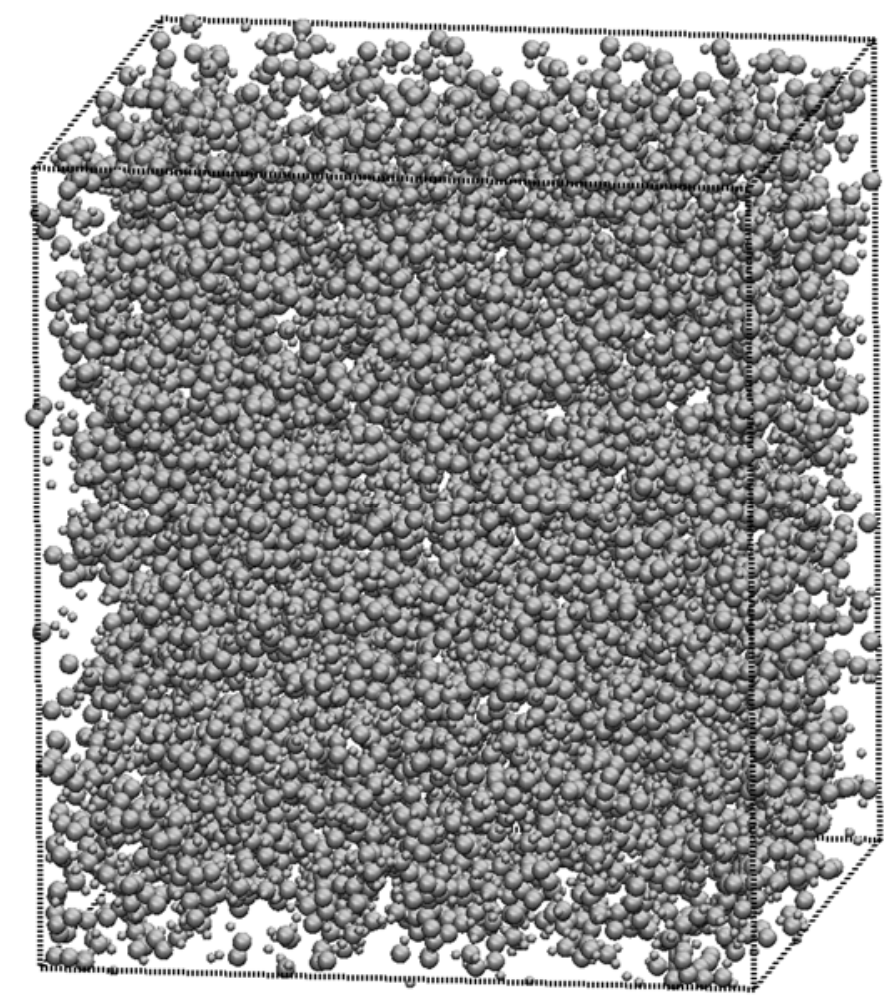

Fig. 8 The atomic unit cell structure for a) crystalline and b) amorphous poly(lactide).

\section{Application of AFEM to crystalline and amorphous poly(lactide)}

Fig. 9 shows the AFEM results for the crystalline structure when a strain is applied in the direction of the polymer chains. An atom on the left hand side of the structure and near the middle $(x=0.129 \mathrm{~nm}, \mathrm{y}=1.17 \mathrm{~nm}, \mathrm{z}=2.757 \mathrm{~nm})$ is fixed in position during the simulations so the structure strains up and down away from the centre. In the simulation a value of $2 \%$ strain is used, although the value of applied strain does not affect the result for Young's modulus. To ensure clarity in the figure atomic displacements are scaled by a factor of 5 . Poisson's ratio can be seen by the translation of the right-most polymer chains in the figure away from the edge of the structure: the structure contracts in the horizontal directions when a vertical strain is applied. The structure was also strained in 
the $\mathrm{x}$ and $\mathrm{y}$ directions to determine the mechanical properties normal to the direction of the polymer chains.

The values of Young's modulus and Poisson's ratio are given in Table 1 and Table 2. Young's modulus is greater in the $z$ direction than in the $x$ or $y$ direction, which is to be expected since in the $z$ direction the polymer chain covalent bonds must deform to allow the polymer chain to elongate. In the $\mathrm{x}$ and $\mathrm{y}$ directions, the polymer chains are connected by weaker nonbonded interactions. The Poisson's ratio values $v_{z x}$ and $v_{z y}$, which indicate the $z$ strains that result from applied $x$ and $y$ strains, are smaller than the other values as a result of the crystal being stiffer in the direction of the chains. Table 1 also details the distribution of interatomic potential energy increases amongst the different AFEM element types, which is referred to as strain energy. The percentages of total strain energy attributed to each element type are shown. Nonbond elements contain the majority of strain energy. This supports the general understanding that strain in polymers is accommodated by deformation of weaker intermolecular bonding rather than of stiff covalent bonds. The strain energy fraction contained in nonbonds is much lower when the strain is applied in the $z$ direction than in $\mathrm{x}$ and $\mathrm{y}$. This is because for an applied strain in the $z$ direction, it is not possible for the main chain elements to avoid strain since the chains are physically elongated in the $z$ direction.

The authors of this paper are aware of two atomic studies regarding the theoretical calculation of Young's modulus for crystalline poly(lactide). Montes de Oca and Ward [14] used the COMPASS force field within the molecular dynamics package Materials Studio while Lin et al. [15] used the ab initio method. Montes de Oca and Ward [14] and Lin et al. [15] find values of Young's modulus in the $z$ direction of $36.0 \mathrm{GPa}$ and 14.1 GPa respectively. The small values of Poisson's ratio found by Montes de Oca and Ward [14] of $v_{z x}=0.07$ and $v_{z y}=0.08$ support the small values found in the AFEM simulations. 
The discrepancies between the values of Young's modulus found by AFEM, by Montes de Oca and Ward [14], and by Lin et al. [15] are due to different crystal structures and force fields being used in the simulations and the different assumptions associated with each method.

The values of Young's modulus found in the AFEM simulations are high compared to values that may be expected experimentally. This can be attributed to the fact that the AFEM simulations are static and therefore represent Young's modulus at a temperature of 0 Kelvin. Nakafuku and Takehisa [16] measured Young's modulus of poly(L-lactide) experimentally and found Young's modulus to almost double as the testing temperature of the sample decreased from $20^{\circ} \mathrm{C}$ to $10^{\circ} \mathrm{C}$ even though both temperatures are well below the glass transition temperature. Also, Brown and Clarke [17] and Ding et al. [1] conducted molecular dynamics simulations on polyethylene at various temperatures from near 0 Kelvin to well above the glass transition temperature. Their results indicate that the value of Young's modulus at 0 Kelvin may be approximately 3-8 times greater than the value near to but below the glass transition temperature. Highly crystalline $(>50 \%)$ rods of poly(lactide) with aligned polymer chains can have Young's modulus values in the region of $6-8 \mathrm{GPa}$ [18-20]. If a single crystal had a Young's modulus of $3 x$ this value, at $\mathrm{OK}$ the Young's modulus in the direction of the polymer chains may be

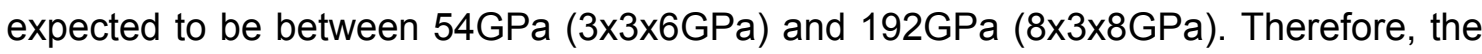
value calculated in Table 1 of $128 \mathrm{GPa}$ is reasonable. 


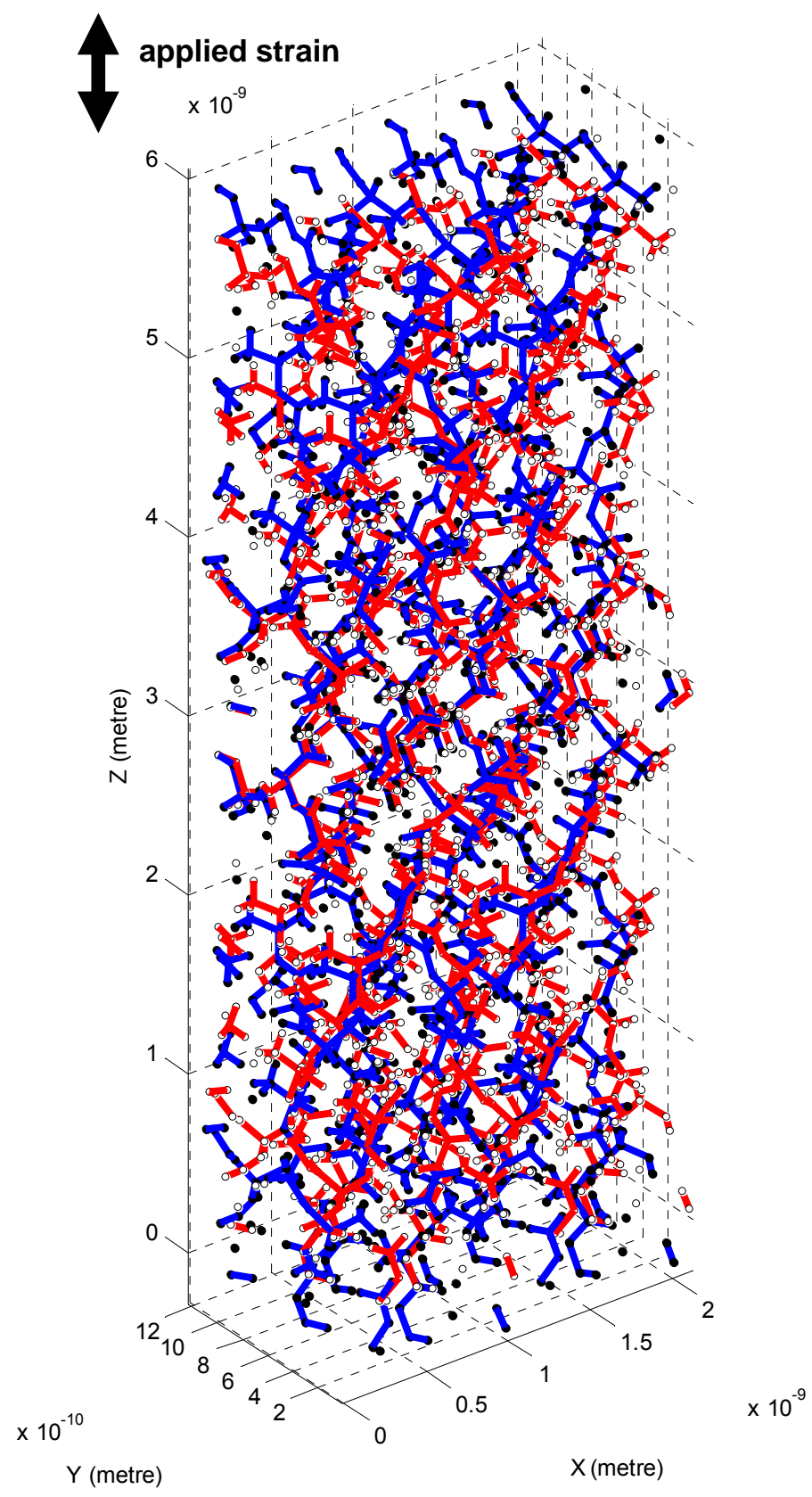

Fig. 9 AFEM results for the crystalline structure. The covalent bonds are shown for the unstrained (red bonds with hollow atoms) and strained (blue bonds with solid atoms) structures.

For the AFEM simulations of the amorphous phase, the values of Young's modulus, Poisson's ratio, and strain energy distribution are given in Table 3. Again, a value of $2 \%$ strain is used. The value of 0.255 for Poisson's ratio is reasonable for a glassy polymer. The value of Young's modulus is higher than typical experimental values (e.g. 0.5-5GPa) which is due to the fact that the simulations are effectively conducted at 0 Kelvin as 
discussed above. In comparison to the crystalline results, amorphous Young's modulus is lower. This result supports the validity of the AFEM simulations because it is to be expected that Young's modulus would be higher in a crystalline phase than in an amorphous phase. As with the crystalline structure, the distribution of strain energy is plausible because the nonbonded interactions contain the majority of strain energy in the AFEM results. The percentage of strain energy in nonbonds for the amorphous structure is in between the percentages for the crystalline structure tested normal to and parallel to the polymer chain orientation. This is because in an amorphous structure, the chains have a variety of orientations.

Typically in experimental publications of biodegradable polymer degradation, the value of Young's modulus is normalised by the initial value, therefore the absolute value is not of critical importance. The primary purpose of the AFEM technique presented in this study is to simulate the effect of chain scission on Young's modulus in the amorphous phase, which is studied in the accompanying paper [11]. This leads to a new mathematical model for Young's modulus degradation in biodegradation polymers.

\begin{tabular}{|l|l|l|l|}
\hline Applied strain direction & $\mathrm{x}$ & $\mathrm{y}$ & $\mathrm{z}$ \\
\hline Young's modulus (GPa) & 50.363 & 53.575 & 128.220 \\
\hline Strain energy in bond-stretch elements & $0.5 \%$ & $1.1 \%$ & $1.9 \%$ \\
\hline Strain energy in bond-angle elements & $1.5 \%$ & $1.6 \%$ & $5.9 \%$ \\
\hline Strain energy in bond-dihedral elements & $0.9 \%$ & $0.8 \%$ & $2.5 \%$ \\
\hline Strain energy in nonbonds elements & $97.1 \%$ & $96.5 \%$ & $89.7 \%$ \\
\hline
\end{tabular}

Table 1 AFEM results for Young's modulus and strain energy distribution in the crystalline structure for an applied displacement in the $\mathrm{x}, \mathrm{y}$, and $\mathrm{z}$ directions. 


\begin{tabular}{|l|l|}
\hline$v_{x y}$ & 0.298 \\
\hline$v_{x z}$ & 0.281 \\
\hline$v_{y x}$ & 0.280 \\
\hline$v_{y z}$ & 0.328 \\
\hline$v_{z x}$ & 0.110 \\
\hline$v_{z y}$ & 0.138 \\
\hline
\end{tabular}

Table 2 Poisson's ratio for the crystalline polymer structure.

\begin{tabular}{|l|l|}
\hline Young's modulus (GPa) & 37.1 \\
\hline Strain energy in bond-stretch elements & $1.9 \%$ \\
\hline Strain energy in bond-angle elements & $4.2 \%$ \\
\hline Strain energy in bond-dihedral elements & $2.0 \%$ \\
\hline Strain energy in nonbonds elements & $91.9 \%$ \\
\hline Poisson's ratio & 0.255 \\
\hline
\end{tabular}

Table 3 AFEM results for Young's modulus, Poisson's ratio and strain energy distribution in the amorphous unit cell.

\section{Conclusion}

A new atomic-scale finite element method (AFEM) was developed for the analysis of biodegradable polymer Young's modulus. The molecular dynamics force field PLAFF2 [5], which was specifically developed for poly(lactide), was used to derive the AFEM technique. In AFEM, atomic-scale finite elements represent the interatomic potential energy functions in molecular dynamics. Three different types of finite elements were required in order to represent potential energy functions (i) for the interatomic separation between two atoms, (ii) for the angle between three atoms that are covalently bonded, and (iii) for the angle between the ijk and jkl planes for four atoms i,j,k,I that are covalently 
bonded. The formulation of these finite elements was described and finite element stiffnesses were derived from the PLAFF2 force field [5]. AFEM simulations were conducted to analyse the mechanical properties of crystalline and amorphous poly(lactide) structures. The strain energy in the strained structures was found to be contained mostly in nonbond elements between atoms that are not covalently bonded. This finding agrees with the general belief that strain is accommodated in between the polymer chains rather than in stiff covalent bonds. The AFEM method enables great computational benefits over iterative molecular dynamics simulations. This allows the repeated analysis of complex amorphous polymer structures. It is therefore ideal for simulating chain scission in biodegradable polymers during degradation. That is the focus of the second study in this two part series [11], which develops a model for the degradation of Young's modulus due to chain scission.

\section{Acknowledgements}

Andrew Gleadall acknowledges an EPSRC PhD studentship and a partial University studentship by the University of Leicester.

The authors would also like to acknowledge James McAliley for supplying the molecular structure coordinate files and PLAFF2 force field files with his thesis [5].

\section{References}

1. Ding, L., R. Davidchack, and J. Pan, A molecular dynamics study of Young's modulus change of semi-crystalline polymers during degradation by chain scissions. Journal of the Mechanical Behavior of Biomedical Materials, 2011. 5(1): p. 224-230. 
2. Wang, Y., et al., An entropy spring model for the Young's modulus change of biodegradable polymers during biodegradation. Journal of the Mechanical Behavior of Biomedical Materials, 2010. 3(1): p. 14-21.

3. Blomqvist, J., B. Mannfors, and L.O. Pietilä, Studies on aliphatic polyesters. Part II. Ab initio, density functional and force field studies of model molecules with two carboxyl groups. Journal of Molecular Structure: THEOCHEM, 2000. 531(1-3): $p$. 359-374.

4. Blomqvist, J., et al., Studies on aliphatic polyesters I: Ab initio, density functional and force field studies of esters with one carboxyl group. Journal of Molecular Structure: THEOCHEM, 1999. 488(1-3): p. 247-262.

5. McAliley, J.H., Development of improved torsional potentials in classical force field descriptions of poly (lactic acid), in Department of Chemical and Biomolecular Engineering. 2009, Clemson University: United States.

6. McAliley, J.H. and D.A. Bruce, Development of force field parameters for molecular simulation of polylactide. Journal of chemical theory and computation, 2011. 7(11): p. 3756-3767.

7. Wang, Y., et al., 2-D nano-scale finite element analysis of a polymer field. Composites Science and Technology, 2003. 63(11): p. 1581-1590.

8. Wang, Y., et al., Atomistic finite elements applicable to solid polymers. Computational Materials Science, 2006. 36(3): p. 292-302.

9. Liu, B., et al., The atomic-scale finite element method. Computer Methods in Applied Mechanics and Engineering, 2004. 193(17-20): p. 1849-1864.

10. Liu, B., et al., Atomic-scale finite element method in multiscale computation with applications to carbon nanotubes. Physical Review B, 2005. 72(3): p. 035435.

11. Gleadall, A., J. Pan, and M.-A. Kruft, An atomic finite element model for biodegradable polymers. Part 2. A model for change in Young's modulus due to polymer chain scission. Submitted for publication. 
12. D. van der Spoel, E.L., B. Hess, A. R. van Buuren, E. Apol, P. J. Meulenhoff, D. P. Tieleman, A. L. T. M. Sijbers, K. A. Feenstra, R. van Drunen, H. J. C. Berendsen, Gromacs User Manual version 4.5.4. 2010, www.gromacs.org.

13. Sasaki, S. and T. Asakura, Helix Distortion and Crystal Structure of the $\alpha$-Form of Poly(l-lactide). Macromolecules, 2003. 36(22): p. 8385-8390.

14. Montes de Oca, H. and I.M. Ward, Structure and mechanical properties of poly(Llactic acid) crystals and fibers. Journal of Polymer Science Part B: Polymer Physics, 2007. 45(8): p. 892-902.

15. Lin, T., X.-Y. Liu, and C. He, Ab Initio Elasticity of Poly(lactic acid) Crystals. The Journal of Physical Chemistry B, 2010. 114(9): p. 3133-3139.

16. Nakafuku, C. and S.-y. Takehisa, Glass transition and mechanical properties of PLLA and PDLLA-PGA copolymer blends. Journal of Applied Polymer Science, 2004. 93(5): p. 2164-2173.

17. Brown, D. and J.H. Clarke, Molecular dynamics simulation of an amorphous polymer under tension. 1. Phenomenology. Macromolecules, 1991. 24(8): p. 2075-2082.

18. Duek, E.A.R., C.A.C. Zavaglia, and W.D. Belangero, In vitro study of poly(lactic acid) pin degradation. Polymer, 1999. 40(23): p. 6465-6473.

19. Nuutinen, J.P., et al., Mechanical properties and in vitro degradation of bioabsorbable self-expanding braided stents. J Biomater Sci Polym Ed, 2003. 14(3): p. 255-66.

20. Pihlajamaki, H., et al., Absorbable pins of self-reinforced poly-L-lactic acid for fixation of fractures and osteotomies. J Bone Joint Surg Br, 1992. 74(6): p. 8537. 